\title{
«Désastres de la guerre» et «embuches de la paix» dans les écrits politiques et historiques d'agrippa d'aubigné
}

\section{Gilles Banderier}

\section{(2) OpenEdition}

Journals

Édition électronique

URL : http://journals.openedition.org/studifrancesi/34097

DOI : 10.4000/studifrancesi.34097

ISSN : 2421-5856

Éditeur

Rosenberg \& Sellier

\section{Édition imprimée}

Date de publication : 1 novembre 2005

Pagination : 303-314

ISSN : 0039-2944

\section{Référence électronique}

Gilles Banderier, « «Désastres de la guerre» et «embuches de la paix» dans les écrits politiques et historiques d'agrippa d'aubigné », Studi Francesi [En ligne], 146 (XLIX | II) | 2005, mis en ligne le 30 novembre 2015, consulté le 18 avril 2021. URL : http://journals.openedition.org/studifrancesi/34097 ; DOI : https://doi.org/10.4000/studifrancesi.34097

\section{(c) (i) (9)}

Studi Francesi è distribuita con Licenza Creative Commons Attribuzione - Non commerciale - Non opere derivate 4.0 Internazionale. 


\title{
DISCUSSIONI E COMUNICAZIONI
}

\section{«Désastres de la guerre» et «embuches de la paix» dans les écrits politiques et historiques d'Agrippa d'Aubigné ${ }^{1}$}

\author{
The paths of glory lead but to the grave. \\ Thomas Gray
}

Envisagée non plus à l'échelle d'une province ou d'un pays, mais dans la longue durée de l'histoire européenne ${ }^{2}$, la période qui s'étend des années 1520 au Traité de Westphalie ne fut qu'une longue guerre de religion, avec des répits ici ou là: naissance et élimination du mouvement anabaptiste, guerres des paysans en Allemagne et dans ses marches, guerres d'Italie et sac de Rome, conflits religieux en France, révolte des Pays-Bas, rivalité entre l'Angleterre et l'Espagne, guerre de Trente Ans: tout nous incite à nous demander si l'expression de «beau XVI ${ }^{e}$ siècle» dont se servent les historiens a finalement un sens. Pendant plus d'un siècle, les tensions religieuses mises au premier plan et les motivations politiques, souvent inavouées mais jamais absentes, ont alimenté guerres intériemes et conflits entre nations. Peut-être ne s'est on jamais avisé que le $\mathrm{XV}^{\mathrm{e}}$ siècle finissant, le fameux «déclin» du monde gothique, ou 1'«automne» du Moyen Age, qui a fait l'objet de tant de discussions, portait également en germe le déclin de la Renaissance. Je laisse à plus compétent ou plus téméraire le soin de se demander si la Renaissance fut, tous comptes faits, autre chose qu'une illusion d'humaniste. Une chose est sûre: la vie d'Agrippa d'Aubigné s'est déroulée au cœur de cette succession de guerres et en a été profondément marquée. Né en 1552, le jeune Agrippa fut élevé par son père et divers précepteurs, dont l'un, Jean Cottin, se prenait pour l'envoyé de Dieu sur terre et, moins prudent que David Joris, finira - à force de le répéter - brûlé vif en 1589. Fin mars ou début avril 1560, Jean d'Aubigné, qui n'avait pas lu nos modernes psychologues, édifia son fils en lui montrant des têtes coupées en train de pourrir sur les murailles du château d'Amboise. C'est ce qu'il restait de conjurés qui avaient comploté contre les Guise. Comme si ce spectacle atroce ne suffisait pas, Jean d'Aubigné promit au jeune garçon de le maudire, même au-delà de la mort, s'il ne vengeait pas «ces chefs plein d'honneur» ${ }^{3}$. Agrippa fut impressionné - on le serait à moins - et la scène des conjurés d'Amboise

(1) «(...) Dieu qui m’a sauvé (...) des désastres de la guerre, des embûches de la paix» (testament, 24 avril 1630; Euvres complètes, éd. Eugène Réaume et François de Caussade, Paris, Lemerre, 1873 , t. I, p. 124).

(2) Se reporter au bel article de PAul Rosset, L'idéologie de croisade dans les guerres de religion au XVIe siècle, «Schweizerische Zeitschrift für Geschichte - Revue suisse d'histoire», XXXI, $\mathrm{n}^{\circ} 2$, 1981, p. 175.

(3) Agrippa d’Aubigné, Sa vie à ses enfants, éd. Gilbert Schrenck, Paris, Nizet-Societé des Textes Français Modernes, 1986, p. 52. 
constituera dans son œuvre un épisode séminal ${ }^{4}$. Deux ans plus tard, le massacre de Vassy marqua le début des guerres de religion en France. D'Aubigné a vingt ans lors de la Saint-Bathélemy, à laquelle il échappe de justesse. Pendant près de quatre décennies, il paiera de sa personne et sera de toutes les batailles (elles seront plus tard évoquées dans ses ouvres historiques ou autobiographiques), ou peu s'en faut, menées par le camp protestant, qui s'identifia naïvement à Henri de Navarre. La conversion du monarque au catholicisme décevra profondément ses anciens coreligionnaires ${ }^{5}$. D'Aubigné entre alors dans une sorte de dissidence, qui ne l'empêchera pas de pleurer sincèrement son ancien compagnon d'armes, assassiné le 14 mai 1610 et de lui consacrer un éloge vibrant à la fin des Tragiques ${ }^{6}$. La régence de Marie de Médicis le place dans une situation difficile et les menées hostiles auxquelles il participe n'arrangeront rien. Il finira par s'enfuir à Genève, en 1620, pour y mourir dix ans plus tard, alors qu'une guerre européenne, qui ne s'appelait point encore la Guerre de Trente Ans, faisait rage hors des frontières helvétiques.

Jusqu'au bout, la guerre fit partie de la vie d'Agrippa d'Aubigné ${ }^{7}$. Il a intitulé un de ses manuscrits «Livre des missives et discours militaires» ${ }^{8}$. Du soldat ou de l'officier, il a parfois la fâcheuse vantardise, même s'il n'est pas dupe', «la guerre consistant en effects, et non point en discours $\rangle^{10}$. Dans ses lettres, il ne manque jamais une occasion de rappeler ses services militaires: «(...) depuis l'an 1567 que j'a y pris les armes $»^{11} ;$ «(...) depuis 60 ans que j'a y veu de la guerre $»^{12}, \ldots$ Dans son testament, rédigé le 24 avril 1630, quelques jours avant sa fin, il songe encore à mourir en soldat:

$\mathrm{Si}$ je suis en lieu où les gens de guerre me veulent porter, je tiens à honneur leur peine et prouver que ma famille a eu l'honneur d'une couverture de velour noir ${ }^{13}$.

On a pu, avec quelque apparence de raison s'étonner que la statue de Montaigne, qui orne son tombeau bordelais, accomplisse non pas le geste de lire ou d'écrire,

(4) Voir l'étude de Gerhard F. Strasser, War and Poetry: personal experience of Renaissance warfare and visual representation of its horrors in the work of Agrippa d'Aubigné, dans Actes du VIIIe congrès de l'AssociationInternationale de Littérature comparée, p. p. B. Köpeczi et G. M. Vajda, Stuttgart, E. Bieber, 1980, t. I, pp. 172-181.

(5) Les relations entre d'Aubigné et Henri IV ont été étudiées, dans des perspectives très différentes, par Jacques Bailbé (L'image de Henri IV dans l'ouvre d'Agrippa d'Aubigné, dans L'image du souverain dans les lettres françaises, actes du colloque de Strasbourg, mai 1983, Paris, Klincksieck, 1985, pp. 27-40; repris en volume dans le recueil Agrippa d'Aubigné, Paris, Champion, 1995, pp. 123-138) er Martine Walzer (Rapports de l'écrit à l'histoire et au "politique": l'Image de Henri IV dans l'œuvre d'Agrippa d'Aubigné, «Albmeana», III, 1990, pp. 109-131).

(6) Voir "À la France delivrée" d'Agrippa d'Aubigné: quelques variantes inédites (Problèmes albinéens), IV, B. H. R., LXII, n³ 3, 2000, pp. 647-651.

(7) Il est frappant de constater que les Mémoires sur la vie d'Agrippa d'Aubighé, qui correspondent probablement à la biographie qu'un certain La Chapelle fut chargé d'écrire par Madame de Maintenon (Bibliothèque municipale de Versailles, manuscrit G. 226, f $6 \mathrm{v}^{\circ}$; Honoré BonHOMme, Madame de Maintenon et sa famille, Paris, Didier, 1863, pp. 231-232), mentionnent à peine l'ecrivain ronfla plus large place à l'homme de guerre (édition critique par mes soins en préparation aux éditions Champion, Paris).

(8) RÉaume, t. I, pp. 131-195

(9) «Il [Biron] disoit que la vanité estoit un cinquiesme element, et celuy des soldats» (lettre à M. de Sainte-Marthe; Réaume, t. I, p. 190) et sa lettre au capitaine Rufigny: «(...) encor que le Mareschal de Biron dict, en montrant son plumet, que la vanité estoit le cinquiesme element des gens de guerre, si est-ce qu'elle a ses differences: car celle qui pousse à plus faire, et plus estre du mestier, sera une honorable vanité: mais celle qui conduict au parestre sans estre, est la peste des gens de guerre» (RÉAUME, t. I p. 194).

(10) Lettre non datée à Saint-Gelais; RÉAume, t. I, p. 114 .

(11) Lettre non datée au marquis de Castelnault et au sieur de Campet; RÉAume, t. I, p. 156.

(12) Lettre non datée à M. de Touverac; RÉAume, t. I p. 179. Dans l'avide «L'Imprimeur au Lecteur» qui ouvre l'Histoire Universelle, d'Aubigné (qui a rédigé ce texte) parle de lui à la troisième personne: «(..) ayant commencé son premier siege dans Orléans 1562 et pourtant esté soldat 54 ans, capitaine 50, Mestre de camp 44 et Mareschal de camp 32 annees, à compter dés l'an MDCXVI datte de la premiere impression [de l'Histoire Universelle]» (Amsterdam [en fait Genève], J. Commelin [en fait Pierre Aubert], 1626, t. I, p. 11 ; éd. A. Thierry, Genève, Droz, 1981, t. I p. 19)

(13) Ed. cit., t. I, p. 124. 
qui nous eût été familier, mais le représente, de manière surprenante, sanglé dans une cuirasse, l'épée au côté et un casque posé près de la tête, tel un gisant médiéval. Marie de Gournay, à qui l'on concédera une certaine familiarité avec l'homme et l'œuvre, s'écriera en manière de réclame, dans sa préface à l'édition de 1595: «Quelle escole de guerre et d'état est-ce que ce livre!» ${ }^{14}$. Mais n'oublions pas que l'auteur des Essais appartenait à la noblesse d'épée, dont la guerre constituait la raison d'être «la forme propre, et seule, et essencielle, de noblesse en France, c'est la vacation militaire», écrira-t-il ${ }^{15}$. Il n'était donc pas anormal qu'on l'immortalisât ainsi. Verra-t-on dans l'activité belliqueuse du roturier qu'était d'Aubigné ${ }^{16}$, une aspiration à la noblesse? Plutôt l'accomplissement d'une promesse et le service d'une cause. L'important est que l'écrivain ne se laisse pas dissocier du guerrier: «bon pour la plume et le poil», disait de lui Brantôme, et un mystérieux thuriféraire, du nom de J. Olivier, écrivait:

Si tost que tu fuz né, d'Aubigny, deux grands dieux

Coururent à l'envi pour voir ta belle face ${ }^{17}$ :

Mars souflla dedans toi la force et le courage,

Apollon le sçavoir, et chant melodieux.

Les palmes, les lauriers, qu'as acquis en maints lieux

Portent de ta valeur suffisant tesmoignage.

Tes doux-doctes escrits, et ton disert langage

Monstrent combien tu as l'esprit ingenieux ${ }^{18}$.

Pas plus qu'on ne peut dissocier l'homme de plume de l'homme de guerre, on ne saurait séparer la vie de l'œuvre. Les ouvrages d'Agrippa sont datés, dans le sens le plus entier du terme, en ceci qu'ils ne se séparent point des circonstances qui ont présidé à leur composition et qu'ils se prêtent par conséquent mal à une approche formaliste.

Cette œuvre, toutefois, est non seulement vaste, mais encore partiellement inédite. Certains ouvrages ont été perdus, sans qu'il faille juger cette perte irrémédiable; d'autres attendent d'être attribués à d'Aubigné; d'autres enfin sont connus des spécialistes, mais n'ont pas encore été édités ${ }^{19}$. A cela, plusieurs raisons: tout d'abord. Agrippa d'Aubigné est un auteur tardif. Même si, selon son propre témoignage, il lisait le français, le latin, le grec et l'hébreu à six ans, même si nous possédons des pièces imprimées de bonne heure, l'essentiel de son œuvre a été rédigé après 1600 . Il avait alors quarante-huit ans. Ensuite, il a passé une bonne part de sa vie d'écrivain à ruser avec les censures et à se dissimuler derrière des pseudonymes variés, voire sous l'anonymat complet, et tous ces masques sont encore loin d'avoir été levés. On ne peut donc pas encore évoquer l'œuvre de d'Aubigné comme on évoquerait celle de Ronsard ou de Montaigne. Il a paru préférable de limiter le propos aux écrits historiques et politiques, qui forment un ensemble cohérent, quoiqu'aux contours encore quelque peu flous. Bien entendu, chez un écrivain aussi entier que l'est d'Aubigné, on distingue mal ce qui relève de l'histoire ou de la théologie et ce qui ressortit propre-

(14) Consulter l'article «Guerre» du Dictionnaire Montaigne, dirigé par M. Philippe Desan, à paraître aux éditions Champion.

(15) Essais, II, 7 (éd. Villey-Saulnier, p. 384).

(16) Se reporter à la mise at essentielle de $\mathrm{M}$. Gilbert Schrenck, Les origines d'Agrippa d'Aubigné, «B.S.H.P.F.», 129, 1983, pp. 489-518.

(17) L'auteur ou le scripteur ont rapidement remarqué que ce vers ne rimait pas: une note en bas de page porte cette correction: «Au second vers:
Coururent pour te voir et en toy leur image».

(18) Bibliothèque Publique et Universitaire de Genève, manuscrit Tronchin 151, f. $2^{\circ}$ r; publié dans Agrippa d'Aubigné et la tradition des poésies liminaires, «Revue des Amis de Ronsard», XV, 2002, pp. 92-93.

(19) Ainsi une étonnante lettre au doge de Venise (Bibliothèque Publique et Universîtaire, Genève, ms. Tronchin 153 , f. $85 \mathrm{r}^{\circ}-88 \mathrm{v}^{\circ}$ ), qui doit être publiée par les soins de M. Jean-Raymond Fanlo. 
ment à la littérature ou à la politique ${ }^{20}$. Les métaphores et les comparaisons guerrières ne sont pas rares dans la poésie amoureuse d'Agrippa ${ }^{21}$, mais il faut faire le départ entre ce qui appartient en propre au chantre de Diane et ce qui relève d'une tradition établie. De même, on a exclu les textes de controverse théologique, qui ne sont que la guerre de religion continuée par d'autres moyens. Soit donc le corpus suivant:

1595-1612: première rédaction de l'Histoire Universelle (publiée à Maillé de 1618 à 1620, puis rééditée à Genève en 1626)

entre 1616 et 1620: le traité sur le Debvoir mutuel des roys et des subjects (compléter l'édition de Réaume par le chapitre inédit publié dans la B.H.R, LXIV, n 2, 2002, pp. 369-376).

1620-1621: Raisonnement d'estat sur la guerre de la Valteline (P.F.S.C.L., XXVIIL nº 54, 2001, pp. 141-151).

Fin 1621: Explication familiere (éd. Jean-Raymond Fanlo, B.H.R, LV, n 3, 1993, pp. 611-644).

1621-1622: Traitté sur la guerre civile (compléter l'édition de Réaume par le texte publié dans le B.S.H.P.F., CXL VII, 2001, pp. 285-303).

1622: «Instruction d'Estat» (texte inédit conservé dans le manuscrit Tronchin 148 de la Bibliothèque publique et universitaire de Genève, f. $168 \mathrm{r}^{\circ}-175 \mathrm{v}^{\circ}$ ).

1622: Docteur d'Hildeberg (éd. Jean-Raymond Fanlo, B.H.R.: LV, n 3, 1993, pp. 611-644) fin 1627: Advis au Roy de la Grande Bretagne (B.H.R, LXI, n² 2, 1999, p. 509-514).

1627-1629: «Abregé d'une harangue faitte au Roy par l'evesque de Maillezays» (French Studies Bulletin, 79, 2001, pp. 4-8).

1628-1629: Advis aux princes chrestiens (B.S.H.P.F., CXLVIII, 2002, pp. 309-335).

On verra dans un premier temps que l'alternance de périodes de conflit et de moments de répit, cette alternance qui est le moteur de l'Histoire, n'est jamais contingente, dans la perspective retenue par Agrippa d'Aubigné. Non seulement Dieu intervient dans le cours des événements humains, mais encore on constatera, dans un second temps, que l'existence de vastes machinations, destinées à assurer la suprématie du catholicisme ou de la Reforme, imprime un sens à l'Histoire. Si des forces mystérieuses sont à l'œuvre, la notion de guerre «juste» finit par acquérir une nouvelle extension, liée au tyrannicide et au devoir de révolte des Protestants opprimés par un monarque injuste.

Le bilan de «60 ans de guerres civiles» en France était impressionnant: les «massacres particuliers et generaux» avaient provoqué «la mort d'un million d'hommes,

(20) Voir les études de Marguerite Soulié, Les idées politiques d'Agrippa d'Aubigné de 1580 à 1590, Les écrivains et la politique dans le SudOuest de la France autour des années 1580, actes du colloque de Bordeaux (6-7 novembre 1981), Bordeaux, Presses Universitaires, 1982, pp. 163 168 et Gilbert Schrenck, Agrippa d'Aubigné et la liberté de conscience d'après ses écrits politiques dans La liberté de conscience - XVI ${ }^{\mathrm{e}}$-XVII ${ }^{\mathrm{e}}$ siècles, actes du colloque de Mulhouse et Bâle (1989), Genève, Droz, 1991, pp. 153-169.

(21) Ainsi dans le Printemps, éd. Henri Weber, Paris, P.U.F., s.d. [1960], pièces X et XCIV (pp. 66-67, 161-162). 
la ruine de plusieurs villes et pais entiers ${ }^{22}$. D'Aubigné n'exagère pas ou, plutôt, il sous-estime la situation: combats, famines, sièges, exactions, épidémies, avaient causé la mort de trois millions de personnes, le quart de la population française. Face à un résultat aussi consternant, le rôle de Dieu, s'il en avait un, aurait pu être passé sous silence avec tact. Mais d'Aubigné est, cela va presque sans dire, un croyant, un homme sûr de sa foi et que n'atteindront jamais les doutes qui assaillaient Job. Pour d'Aubigné comme pour des millions de personnes avant et après lui, Dieu intervient dans l'Histoire. Rien n'est contingent et la guerre n'échappe pas à ce principe. Nous sommes loin du «tale / Told by an idiot, full of sound and fury, / Signifying nothing» de Macbeth. Dans la sublime apocalypse des Tragiques, Dieu intervient de manière directe, descendant sur terre pour se faire le témoin des horreurs commises, avant de décider de mettre un terme à l'Histoire, telle que les hommes l'avaient connue. D'Aubigné ne pouvait point le faire se manifester aussi directement dans l'Histoire Universelle et il était impossible de présenter les faits collectés selon un schéma préétabli trop apparent. Ces considérations n'interdisaient pas à l'ensemble d'être placé sous le regard de $\mathrm{Dieu}^{23}$ :

(...) acceptez la peinture d'un temps calamiteux, plein d'ambitieux desseins, de fidelitez et infidelitez remarquables, de prudences et temeritez, de succes heureux ou malheureux, de vertus relevées et d'infames laschetez, de mutations tant inesperees, qu'aisement vous tirerez de ces narrations le vrai fruict de toute l'Histoire, qui est de connoistre en la folie et la foiblesse des hommes, le jugement et la force de Dieu ${ }^{24}$.

Les signes divins tendent donc à se fair discrets, beaucoup plus discrets, en tout cas, que chez d'autres historiens. Dans les Mémoires de la Ligue, colligés par le pasteur Simon Goulart, on lit qu'en la bataille d'Ivry, si «celebre et memorable», «il s'est peu reconoistre (...) deux ou trois choses si extraordinaires, qu'ils ont deu faire juger, que la main de Dieu y a voulu grandement opérer» ${ }^{25}$. Chez d'Aubigné, le récit de cette bataille est entièrement sécularisé ${ }^{26}$. Narrant la bataille de Coutras, livrée et gagnée en deux heures, l'historien commence néanmoins par nier une intervention surnaturelle, pour mieux la souligner ensuite: «Plusieurs ont trouvé et descrit le gain de cette bataille comme miraculeux; je n'en juge pas ainsi (...). Mais ce qu'on peut trouver estrange, c'est que les victorieux n'eurent de perte que çinq gentilhommes et vingt soldats. Les vaincus y laisserent plus que quatre cents gentilshommes, ou personnes qui tenoient ce rang, plus de 3000 hommes de pied; telle quantité de noblesse ne s'estant perdüe aux trois plus sanglantes battilles de ce siècle ${ }^{27}$. Les signes divins

(22) Histoire Universelle, éd. de 1626, t. I, col. 50-51; éd. A. Thierry, t. I, p. 129.

(23) Je reprends ici des vues formulées dans une étude sur Henri IV et la paix: Du Bartas, d'Aubigné, Jeannin dans Paix des armes, paix des âmes, actes du colloque international de Pau (8-11 octobre 1998), p. p. P. Mironneau et I. Pébay-Clottes, Paris, Imprimerie Nationale, 2000, pp. 381-394.

(24) Histoire Universelle, 1626,. t. I, col. 7; éd. A. Thierry, t. I, p. 10. D'autres affirmations vont dans le même sens: «(...) qu'en cerchant la gloire de ce precieux instrument [Henri IV], ils [les miens] ayent pour but principal celle du bras qui l'a desployé, employé, et ployé quand il lui a pleu. Car toutes les loüanges qu'on donne aux Princes, sont hors d'œuvre et mal assises, si elles n'ont pour fueille et fondement celle du Dieu vivant, à qui seul appartient honneur et empire à l'eternité» (préface, éd. de 1626, t. I, col. 10; éd. A. Thierry, t. I, p. 16); «(...) les succés envoyent par force les yeux et les esprits de la terre tenebreuse au Ciel luisant, des splendeurs qui passent aux eternelles, des Royaumes caduq au permanent, et en fin de ce qui paroist estre vivre et regner, à ce qui seul est, vit, et regne veritablement» («Appendix», éd. de 1626, t. III, col. 743; éd. A. Thierry, t. IX, p. 412).

(25) Mémoires de la Ligue, édition de 1595, t. IV, p. 225 et 270.

(26) Histoire Universelle, éd. de 1626, t. III, 1. 3, chap. 6, col. 315322; éd. A. Thierry, t. VIII, pp. 164-173, et la note 1, p. 164.

(27) Éd. cit., éd. de 1626, t. III, 1. I, chap. 18, col. 89; éd. A. Thierry, t. VII, p. 143. 
se fondent surtout dans l'arrière-plan de l'Histoire et dans la dispositio même de cette somme. En effet, d'Aubigné rend compte du plan de sa grande œuvre en ces termes:

Le second Tome entre un peu devant la S. Barthelemi, et acheve aux premiers exploits apparens de la Ligue, où commence le Troisieme pour se reposer au grand repos de la France, quand Henri le Grand s'est veu paisible roi ${ }^{28}$.

et précise: «Chacun de ses Livres finit par une fin de guerre, ayant pour sa borne un Edict de Paix, ou chose equipolente ${ }^{29}$. Tout au long de l'ouvrage, on observe une forme de «respiration» de l'Histoire, de mouvement pendulaire entre la guerre et la paix, mouvement qui semble atteindre la stase avec les différents traités signés entre 1598 et 1601 . Néanmoins, dans l'appendice au tome III, d'Aubigné s'attarde sur le projet que caressait Henri IV d'attaquer l'Espagne. Nous savons qu'au moment de son assassinat, le Bourbon mettait sur pied une des plus grandes armées jamais constituées, dans le but précis d'envahir le royaume de leurs Majestés très catholiques. Avec cette nouvelle guerre et, peut être, la nouvelle paix qui la clora ${ }^{30}$, le cycle paraît reprendre.

Cette invasion de l'Espagne fait partie d'un projet plus vaste. L'idée d'un «grand dessein» obsédait littéralement Agrippa d'Aubigné. Jean-Raymond Fanlo a montré comment le projet annoncé par Sully s'est transformé, dans l'Histoire Universelle, en croisade impériale $^{31}$. On trouve dans ses papiers ${ }^{32}$ un petit dossier qui expose en détail un projet mêlant le Pape, l'Empereur et le roi d'Espagne, visant à déraciner l'hérésie protestante. Toutes les guerres particulières ne sont menées qu'en fonction de cet objectif général et ultime. La mention de Marie Stuart, alors encore en vie, montre que ce texte a été rédigé avant $1587^{33}$. Même si d'Aubigné n'en est pas l'auteur, le fait qu'il l'ait conservé montre qu'il y attachait quelque importance et, sans doute aussi, quelque crédit. Cette idée de complot a été suggérée de bonne heure, puisque le cardinal de Granvelle en est désigné comme un des instigateurs.

Plus on avance dans le nouveau siècle et plus le «grand dessin» paraît se préciser. En effet, la France des derniers Valois et du premier Bourbon avait réussi à ne pas se laisser enfermer dans l'étau des Habsbourg. Les deux premières décennies du XVII siècle voient au contraire le cercle se reformer lentement. L'année 1620 marque un tournant et semble inaugurer la première phase d'un plan européen pour convertir le continent, lorsque l'Espagne envahit une petite vallée des Alpes, la Valteline, de

(28) Ibid., «L'Imprimeur au Lecteur», éd. de 1626, t. I, col. 11; éd. A. Thierry, t. I, p. 17.

(29) Ibid., loc. cit. Ce qui donne, au tome I, 1. I, ch. 28, «Articles de la paix d'Espagne»; 1. II, ch. 32, «De la Pai. Edict de janvier»; 1. III, ch. 26, «Premier Edict de paix»; 1. IV, ch. 22, «Paix des guerres civiles»; 1. V, ch. 33, «De la Paix». Au tome II: 1. I, ch. 20, «De la Paix d la Rochelle, cinquiesme en nombre»; 1. II, ch. 27, «De la Paix qui prit le nom de Monsieur»; 1. III, ch. 26, «De la Paix septiesme»; 1. IV, ch. 22, «Conclusion de la Paix» ; l. V, ch. 23, «De l'Edit de Juillet. Prerniere paix avec les Ligues». Au tome III: 1. I, ch. 29, «Paix avec les Liguez, Edict de Juillet»; 1. II, ch. 31, «Declaration du Roi de Navarre pour le tresve»; 1. III, ch. 341, «Premiere paix des Liguez»; 1. IV, ch. 29, «Derniere paix des Liguez»; 1. V, ch. 20, «Articles abregez de la derniere paix, des guerres civiles de France jusques à l'an 1601».

(30) Serait-elle marquée par l'établissement d'une monarchie universelle d'essence française?

(31) Ibid., «Appendix», éd. A. Thierry, t. IX, pp. 404-405; J.-R. FANLO, Croisade et millénarisme dans l'œuvre d'Agrippa d'Aubigné dans Formes du millénarisme en Europe à l'aube des temps modernes, actes du colloque de Marseille (10-12 septembre 1998), ed. Jean-Raymond Fanlo et André Tournon, Paris, Champion, 2001, p. 401.

(32) Genève, Bibliothèque Publique et Universitaire, manuscrit Tronchin 142, f. $72 \mathrm{r}^{\circ}-74 \mathrm{r}^{\circ}$; publié dans Agrippa d'Aubigné et le "grand dessein": un document inédit, «P.F.S.C.L.» XXX, n 58, 2003, pp. 197-207.

(33) On rencontre dans la correspondance de Théodore de Bèze de fréquentes allusions au syncretismus ou complot papiste (lettre de Bèze au comte Ludwig von Sayn, Genève, 14 mars 1583, Correspondance, éd. Alain Dufour et alii, Genève, Droz, 2002, t. XXIV, p. 72; lettre de Gwalther à Bèze, Zurich, 15 mars 1583, ibid, t. XXIV, p. 78). 
prime abord dépourvue d'importance ${ }^{34}$, en réalité passage du Nord au Sud, reliant les possessions italiennes de l'Espagne aux Pays-Bas. Une copieuse production pamphlétaire est consacrée à cette invasion et plusieurs brochures vulgarisent l'idée d'un grand dessein prêté à l'Espagne. L'une d'elles évoque les «pernicieux desseins qu'ils [les Espagnols] ont de les [les églises réformées] exterminer toutes, lors que leurs satellites et armées profanes seront prestes pour les executer, et ainsi que leur project en a esté faict en leur generale conjuration forgée depuis quelques années en çà par l'artifice des Jesuittes: dans la boutique Vaticane de Rome comme il a esté certifié par hommes dignes de foy qui se sont trouvez dans le conseil de ces Judaiques Romains» ${ }^{35}$. Une autre commence ainsi: «Les Espagnols pour tousjours advancer leur Monarchie universelle, de laquelle ils ont jetté les fondemens dés le terms et sous l'Empire de Charles Quint, et n'en demordent jamais le dessein, ont si bien et heureusement travaillé depuis dix ou douze ans en plusieurs endroits de l'Europe, et mesme és environs de la France, qu'il ne meur reste que fort peu à conquerir pour nous environner de tous costez, et se rendre peu à peu maistres et arbitre de la Chrestienté» ${ }^{36}$. Même l'ambassadeur Bassompierre n'échappe pas à la suspicion ambiante: «Ce fut lors que les Espagnols virent quelque jour au dessein (...) de s'approprier la Valteline, qui leur est utile et necessaire (...) au project de leurs futures conquestes, pour parvenir à la Monarchie universelle» ${ }^{37}$. À partir de 1620, les allusions au «grand dessein» se multiplient frénétiquement sous la plume d'Agrippa:

«Vous savez mieux que moy (...) comment en la conjuration, union et contribution de toutes les parts de l'Europe occidentale, tout s'ameute à la destn1ction des fideles: et si c'est avec divers pretextes et moyens differents, tout aboutist à mesme point $\gg^{38}$.

«Venons à la cause d'estat principale motrice de ces agitations: cause la plus certaine et la moins cognue: cause qui par la playe d'une partie put aisement gangrener tout le corps. C'est le grand dessein que les Jesuites ont projetté dez leur advenement au monde, assavoir d'eslever l'Espaignol par dessus toutes les puissances seculieres de la terre, et le rendre en effect Roy

(34) Et de dimensions fort réduites: environ 90 kilomètres de long sur 3 ou 4 de large. «(...) ceste Valteline est une des plus agreables et delicieuses valées que les yeux de l'homme puissent voir dans l'Europe. Elle est située entre deux hautes montagnes. Elle est abondante en vins forts et genereux, qui se transportent bien loin: fertile en excellents fruits, bleds et pasturages, traversée de belles rivieres», écrit Anton de Molina dans la préfàce (pp. 34) au précieux recueil où il réunit divers opuscules relatifs au conflit: LA // VALTELINE, // OU MEMOIRES, // DISCOURS, TRAICTEZ, // ET // Actes des Negotiations sur le sujet des troubles \& // guerres survenues en la Valteline \& au pays des // Grizons, depuis l'invasion \& usurpation de ladite // Valteline en l'an 1620. jusques en l'an 1629. [...] // [...] // Recueil tres-utile \& necessaire en ce temps à tous // bons Patriotes. // [vignette] // A GENEVE. // [filet] // M.DC.XXXI.

(35) EXHORATION // ET // ADVIS AUX EGLI- // SES DE LA RELIGION // REFORMEE, // TOUCHANT LE PI- // TOYABLE ET CRUEL // MASSACRE FAIT AUX DEUX EGLISES // reformees de Tell \& Tiras, en la Valtuline $[\ldots] / / 9$ juillet $1620 / /[\ldots] / /$ [fleuron] // A CHOUR, // PAR JEAN BENOIST. // M. DC XX. [p. 3]. Un autre opuscule signale que « (...) le Roy Catholique peut entrer par cette porte [la
Valteline] et par ces Alpes à la domination generale, et effectuellement former la Monarchie universelle par lui pretendue dès long temps, et à laquelle il se va acheminant tantost d'un costé tantost de l'autre, si par le contrepoids de la Monarchie Françoise n'y est remedié de bonne heure» (DISCOURS // SUR L'ESTAT // LAMENTABLE // AUQUEL SONT RE- // DUITES LES TROIS // Ligues des Grizons. // Contre leurs anciennes libertés, \& le traité // de Madrid, fait entre les deux Ma- // jestez le 15 Avril 1621. // [...] // [fluron] // M.DC.XXII. [p. 20]; réimprimé dans le recueil d'Anton de Molina, 1631, pp. 271-272).

(36) PROGREZ // DES // CONQVESTES // DV // ROY D'Espagne // ET MAISON D'AVSTRICHE // en Allemagne, Suisse, Grisons, Italie, // \& frontieres de la France, depuis // la mort du Roy HENRY // LE GRAND. // [fleuron] // M. DC. XXIII. [Bnf, Lb36 2159, p. 3]. Réédité sous un titre plus explicite encore: Dessein perpetuel des Espagnols à la Monarchie universelle, avec les preuves d'iceluy [Bnf, Lb36 2160].

(37) Ambassades du nmreschal de Bassompierre en Espagne l'an 1621, Cologne, Pierre du Marteau, 1668 , p. 2.

(38) Lettre à d'Arsens, 1621, Euvres complètes, éd. Réaume et Caussade, t. II, p. 132. 
Catholique c'est à dire Tyran universel de toute la Chrestienté, ains de tout le monde» (texte destiné à Louis XIII et rédigé entre août et novembre 1621)»”.

«(...) tout ce qui ensanglante l'Europe aujourd'huy n'est provenu d'aucune colere prise de nouveau, mais d'un long et premedité desseing. (...) il paroist que tout tend à ce qui s'appelle le Grand desseing (...). Ce grand dessein est de reduire l'Europe soubs un seul Evesque et soubs un seul Roy, tousjours le titre de la religion le premier en paroles et le moins exprés és choses; et tout de mesme que le voile spirituel s'accomode à son dessous, vous voyez encore deux autres branches couvertes l'une de l'autre à sçavoir soubs l'extinction et dissipation de l'heresie à l'avantage du Siege Romain, la destruction de toutes les Républiques au profit des Souverains ${ }^{40}$.

L'Apocalypse approche à grands pas: «Voicy donc le temps arrivé, où les ruses de l'Ante-Christ se desployent en fureurs» ${ }^{41}$. On n'aurait aucune peine à multiplier les citations et l'on se tromperait, assurément, en imaginant que Bassompierre, d'Aubigné et quelques autres ont succombé à une sorte de paranoïa collective. Le «grand dessein» donnait un sens global à des événements isolés. Tous les petits conflits locaux, éparpillés, se trouvaient ainsi reliés à un ensemble plus vaste et l'invasion de la Valteline, par exemple, prenait une portée profonde. Une chose pouvait être tenue pour sûre: la Contre-Réforme gagnait du terrain:

Vous voyez, Monseigneur, quel est le visage de l'Europe entiere, espouventable de 34 grandes armées, sur lesquelles le ciel gresle, et faict plus de meurtres justes que d'injustes: le couteau, la faim et la peste marchent au son des tambours, et font leurs charges plus souvent que les trompettes ne la Sonnent. L'Italie, l'Almagne, la France et les Pays-Bas sont puants de morts, et plus que les charougnes y puent les defections, les infidelitez et le mespris de toute vertu, en un temps où elle feroit tant de besoin ${ }^{42}$.

La fureur des peuples est en un tel penchant, que nulle bride ne les pourroit relever du precipice, et on trouvera que ls orages de Royaume en auront pourri les reines, qui se rompront quand on voudra tenir la main haulte, et garantir la cheute dans le malheur ${ }^{43}$.

A peine quelques mois après l'invasion de la Valteline, l'assemblée de La Rochelle, ouverte le 25 décembre 1620, manifesta dès exigences qui parurent délirantes, eu égard à la situation du parti protestant français. Au printemps de 1621, Louis XIII partit en campagne dans le sud de la France pour attaquer les places fortes du parti, qui s'empressèrent de capituler ${ }^{44}$. Passant à Saumur, le Roi humilia Philippe Duplessis-Mornay ${ }^{45}$, avant de rencontrer à Saint-lean-d'Angély une première poche de résistance, qui tint un mois ${ }^{46}$. Il poursuivit son expédition punitive sans se battre

(39) En marge du 'Traitté sur la guerre civile': un texte inédit d'Agrippa d'Aubigné, «B.S.H.P.F.», CXLVII, 2001, p. 293.

(40) Traitté sur la guerre civile, 1622, ch. IV; RÉAume, t. II, pp. 14-15. Cf. sa remarque à Cavassa, ambassadeur de Venise: «(...) jamais Monarque ne fut bon support des Republiques» (Réaume, t. I, p. 242). Sur les circonstances de la composition de ce traité, voir l'article de Ian Macdonald («Three pamphlets by Agrippa d'Aubigné. Manuscripts and dates of composition», French Studies, XIV, janvier 1960 , pp. 38-51).

(41) Traitté sur la guerre civile, ch. VI; RÉAumE, t. II, p. 27. 403.

(42) Lettre à Rohan, sans date; Réaume, t. I, p.

(43) Lettre au gouvernement de Berne, 1622; Réaume, t. I, p. 246).
(44) «(...) le Roi part de Paris, non pour commencer une guerre, mais pour jouir d'une victoire assurée» (Henri de Rohan, Mémoires sur les choses advenues en France depuis la mort de Henri-leGrand jusques à la paix faite avec les Réformés au mois de juin 1629, éd. Petitot, Collection des mémoires relatifs à l'bistoire de France, 2ème série, 1822 , t. XVIII [=t. I], p. 185).

(45) «Le premier manquement de parole fut pour Saumur, ôté au Plessis-Mornay contre la foi de son écrit» (Mémoires, éd. cit., t. I, p. 185). D’Aubigné évoquera ailleurs «la prise de Saumur, pris de mauvaise grace, estant offert trop gracieusement» (Traitté sur la guerre civile, ch. II; éd. cit., t. II, p. 5).

(46) François de Vaux de Foletier, Le siège de La Rochelle (1931), La Rochelle, Quartier Latin - Rupella, 1978, p. 27. 
trop souvent puisque, à l'exception de Clairac ${ }^{47}$, les villes préférèrent ouvrir grand leurs portes. L'armée royale se trouva enfin, le 17 août 1621, devant Montauban, laquelle n'entendait pas se rndre. Commentant cette campagne, d'Aubigné, qui avait toujours respecté le fils de Henri IV, perdit une partie de ce respect dans un petit texte, demeuré inédit, où il lui écrit:

(...) c'est bien ung chemin plus court pour acquerir le paradis et à moindres frais que celuy que Saint Loys vostre ancestre alloit chercher en Egipte et en Barbarie, là où les incommodités d'un sy loingtain voyage et changemens d'air sy contraire au sien naturel le firent mourir, luy et presque toutes ses gens: $\mathrm{O}$ qu'il vous est bien plus commode de faire la guerre aux infidelles de Poitou et de Languedoc qu'à ceux de Palestine; vous ne sortez point de vostre air et de vostre climat, et quand ils seront exterminés il ne vous manquera point de subject pour vous croiser et guagner ce grand tresor d'indulgences, l'Angleterre, la Hollande, Dannemarc, Suede, Geneve et les Suisses vous en fourniront assés ${ }^{48}$.

\section{In cauda venenum, il conclut:}

Au surplus faittes tout ce que le roy d'Espagne voudra et que le pape vous commandera et vous ne pourrez faillir de vous immortaliser dans l'histoire et de vous acquerir ce saint nom de Louys le simple tant recommandé par nostre Seigneur à ses apostres et quittés ce nom prophane et presumptueux que vos flatteurs vous ont imposé de juste, qui n'apartient qu'à ung seul Dieu ${ }^{49}$.

D’Aubigné correspond avec Mansfeld ${ }^{50}$. Un an plus tard, la prise de Heidelberg, le 19 septembre 1622, plonge les Réformés dans la consternation. Dans un pamphlet intitulé Le Docteur d'Hildeberg, d'Aubigné propose vainement la mise en place d'une ligue protestante. En 1624 Madrid projettera une ligue des Habsbourg, que le pape et les états catholique étaient invités à rejoindre. D'Aubigné avait bien compris que la guerre européenne, qui faisait de la Suisse un havre de paix au milieu d'un continent livré au chaos ${ }^{51}$ (un rôle qu'elle retrouvera sovent par la suite), était - comme tous les autres conflits qui l'avaient précédée - une guerre de religion: «ceste guerre est de religion, qualité qui la rend tresardante, lui hausse le poux, et lui cause des paroxismes du tout estranges» ${ }^{52}$. Les motivations de cette conflagration ne font pas de doute:

Venons à la cause d'estat principale motrice de ces agitations: cause la plus certaine et la moins cognue: cause qui par la playe d'une partie peut aisement gangrener tout le corps. C'est le grand dessein que les Jesuites on projetté dez leur advenement au monde, assavoir d'eslever l'Espaignol par dessus toutes les puissances seculieres de la terre, et le rendre en effect Roy Catholique c'est à dire Tyran universel de toute la Chrestienté, ains de tout le monde. Ce dessein

$(47) \ll(\ldots)$ rin ne fit mine de résister que Clérac, qui étoit bien fortifié et bien muni, et y avoit trois mille hommes de guerré, y compris les habitans» (Rohan, Mémoires, éd. cit., t.1, p. 189). Cf. Le Docteur d'Hildeberg (B.H.R, LV, n' 3, 1993, p. 632).

(48) «Abregé d'une harangue faitte nu Roy par l'evesque de Maillezays», Bibliothèque Publique et Universitaire de Genève, manuscrit Tronchin 148, f. 120 r $^{\circ}$; publié dans Agrippa d'Aubigné et Louis XIII: un texte inédit, «French Studies Bulletin», 79, 2001, pp. 4-8.

(49) Ed. cit., ms. Tronchin 148, f. $120 \mathrm{v}^{\circ}$ (Agrippa d'Aubigné et Louis XIII: un texte inédit, «French Studies Bulletin», 79, 2001, p. 8).

(50) Sur les rapports de d'Aubigné avec le grand guerrier, voir la lettre à Graffenried et Spietz (1622), Réaume, t. I, p. 149; Sa Vie à ses ehfants, éd. G. Schrenck, pp. 205-206 et la lettre de Mansfeld à Agrippa, envoyée le 20 octobre 1621 ('Analecta Albineana' Documents inédits relatifs à Agrippa d'Aubigné, B.S.H.P.F., 2003).

(51) «(...) l'Europe entiere (à commencer dés le bout de la Moscovie, jusques au miserable Portugal) est puante de leurs meurtres, poudreuse de leurs ruines, et fumeuse de leurs embrasements» (Le docteur d'Hildeberg, B.H.R., LV, n 3, 1993, p. 630).

(52) Bibliothèque Publique et Universitaire de Genève, manuscrit Tronchin 146, f. 233 v $^{\circ}$; B.S.H.P.F., CXLVII, 2001, p. 289. 
a enfanté ci devant le grand e effroyable monstre de la Ligue que l'invincible Anthree Henry le grand estouffa dez sa naissance. Ce mesme dessein faict aujourd'huy entrechocquer tous les peuples de l'Europe afin que ce fier Nemrod puisse bastir une Babel du debris des souverainetés dont il ne scauroit venir à chef si elles demeuroient en leur entier ${ }^{53}$.

Dans deux textes plus tardifs, adressés l'un à Charles ${ }^{\text {er }}$ d'Angleterre (1627), l'autre à l'ensemble des «Princes chrestiens» (1628-1629), d'Aubigné souligne nettement le rôle joué par les Habsbourg:

Toutte l'Europe est divisé en deux Religions, l'une et l'autre ne croyant avoir de veritable et asseurée protection aux choses du monde qu'en la Maison d'Austriche et en celle de la Grande Bretaigne. De ceste opinion ceulx de la Maison d'Austriche ont faicts les fondaments pour parvenir à la Monarchie Universelle ${ }^{54}$.

Les deux grandes puissances qui tiennent en contrepoids les autres Princes de la Chrestienté, sont les maisons de France et d'Austriche ${ }^{55}$ : car ce sont les seules qui peuvent sans secours d'autruy faire la guerre, et l'entretenir tant qu'elles veulent, ne manquants pour cet effet d'homes ny d'argent. (...) La premiere n'a dessein que de se maintenir. La seconde a celuy de s'accroistre. La premiere ne veut la guerre qu'à l'extremité, et selon qu'elle y est forcée par la necessité de sa conservation, ou de celle de ses voisins. La seconde a tousjours les armes à la main pour empieter quelque chose sur l'autruy. La premiere tasche à maintenir en union ses voisins, pour les faire subsister. La seconde d'y semer de la division, pour y profiter. (...) Bref la premiere se contente de maintenir ce qu'elle possede: et la seconde aspire à la Monarchie universelle $e^{56}$.

Ce qui distingue ces deux textes, par ailleurs fort proches, est la question de savoir à qui s'adresser pour s'opposer aux prétentions des Habsbourg. A la France ou à la Grande-Bretagne? Tout dépend de l'heure. Dans les deux cas de figure apparaît la notion de guerre «juste». Dans une lettre du 29 mars 1616, d'Aubigné écrivait à son correspondant, hélas demeuré anonyme: «J'ay toujours estimé que toute guerre qui n'a pour but la paix est injuste et, de là, malheureuse. Mais plus inique est encores la paix qui a la guerre pour but $\gg^{57}$. Rien n'est plus légitime que de prendre les armes contre un envahisseur étranger ou contre un monarque qui méprise la parole donnée à ses sujets. D’Aubigné a consacré un traité spécial à la question du contrat entre le prince et son peuple (contrat dont la rupture peut conduire au tyrannicide), le Debvoir mutuel des roys et des subjects. Dans un chapitre inédit, publié récemment, il écrit:

Il ne s'agit pas de sçavoir s'il faut esmouvoir la guerre à un prince ou luy envahir ses terres ou se soustraire de ses commendements en toutes choses qui ne nous rendent point coulpables devant Dieu, ny relasche rien du respect et reverence qui luy est deue par la loy de Dieu, mais il s'agist si un Roy se laissant aller aux desirs de quelques boutefeux et meurtriers, engence du diable meurtrier des le commencement ${ }^{58}$, et commendoit l'extermination entiere et cruelle de plusieurs milliers de ses sujects voire la ruine de toute la liberté et honnesteté de son Royaume,

(53) Ibid., f. $235 \mathrm{r}^{\circ} / \mathrm{v}^{\circ}$; B.S.H.P.F., CXLVII, 2001, pp. $293-294$.

(54) «Advis au roy de la Grande Bretagne», British Library, Additional Manuscript 48101 (anciennement ms. Yelverton 110), f. 394 r ; B.H.R, LXI-2, 1999 , pp. 509-510.

(55) Comparer ce passage avec le Docteur d'Hildeberg («Les uns exaltent la maison d'Austriche, les autres celle de France, ou bien toutes les deux à la fois, preschant devant les plus foibles en termes expres la splendeur, les richesses, les honneurs qui descoulent des Monarchies», B.H.R, LV, n ${ }^{\circ} 3$, 1993, p. 638).

(56) Bibliothèque Publique et Universitaire, Genève, manuscrit Tronchin 148, pp. 1-3 (texte imprimé); B.S.H.P.F, CXLVIII, 2002, pp. 321-322.

(57) Pages inédites, éd. cit., p. 29.

(58) «C'est tousjours du levain de ce vieil serpent meurtrier dés le commencement du monde» (Traité sur les guerres civiles, ch. VI, RÉAUME, t. II, p. 30). 
voire encor de luy mesme, si dire le grand nombre des subjects contre lesquels s'adresse la fureur du prince pour les exterminer, peut opposer un bouclier pour empescher ce grand mal ${ }^{59}$.

On peut donc user de violence envers son souverain sans désobéir à Dieu. De même, une guerre sera juste dés lors qu'elle vengera une injustice et constituera le dernier recours des protestants menacés par les catholiques ou des Français attaqués par l'Espagne, ainsi que d'Aubigné l'écrit dans un texte vraisemblablement destiné à Louis XIII:

Ceulx qui font profession de juger et prononsticquer par quelques signes les fms et procedez d'un estat voiant ces demieres années le vostre si miserablement tourmenté de ceste mauldite hayne qui jadis emporta plusieurs empires, monarchies de ce monde, et encore aujourd'huy en travaille une bonne partie, ne pouvant autre chose juger synon que la contrarieté des humeurs en aigrira par les longues années les recheutes et autres redoublés ne finiront jamais que par la fin de vostre pauvre estat dont la plus part l'avoient abandonné et ceulx qui mieux esperoiem craignoient qu'au moings il y deust coupper une partie ou de ses membres ou de sa force. Maintenant, sire, Dieu comme le seul medecin qui en ceste maladie desesperé pouvoit donner remede, faict tant de grace à vostre majesté que de le veoir gary, refaict sur piedz. Reste de le preserver d'une recheute et le maintenir par tous moiens en sant loisible. Et a cecy n'est rien plus propre qu'un exercice pas à temps qui consomme les mauvaises humeurs qui les pourroient causer et confinner.

Ce qui entretient la santé est d'entreprendre une guerre abhorée pour entretenir la paix dedans le royaume. Tous bons politiques ont tout temps faict mectre ung ennemy en teste à ung peuple aguery de peur qu'il ne devienne ennemy de soy mesme. Chacun sçait comme le François, qui a gousté ces armez les peut et comme souvent de gaieté de cœur par faulte d'ennemy il querelle son compaignon et amy mesme. L'Itallien, l'Allemant, le Suisse, la paix faicte, retourne à son metier. Le François deprist tout autre metier et demeure soldat par faulte de plus commode exercice, plusieurs aimant mieulx rechercher la guerre au loing ou la faire aux passans sur les chemins, que de se reposer chez eul $x^{60}$.

Même si d'Aubigné n'avait sans doute pas sous les yeux ces textes fondateurs que sont le décret de Gratien ou la Summa decretorum de Rufin, il n'en développe pas moins une conception de la guerre «juste» qui n'est pas très éloignée de celle des théoriciens médiévaux. L'idée de croisade est fort présente chez lui: croisade de la France contre la puissance espagnole ou, plus inquiétante - de son point de vue - croisade des puissances catholiques coalisées pour transformer la Réforme européenne en un lointain souvenir. D'Aubigné est naturellement convaincu de se trouver dans le bon camp et de mener une guerre juste, qui se reconnaîtra à un certain nombre de signes, de victoires ${ }^{61}$ et qui autorisera des moyens extraordinaires. Il approuve hautement l'assassinat de Concini ${ }^{62}$. Dans un texte composé après 1625. Agrippa d'Aubigné recommandera l'emploi de solutions qu'il eût repoussées avec indignations quelques années plus tôt, en les couvrant de l'épithète infamante de «machiavélien» ${ }^{63}$. Dans l'Advis adressé à Charles $I^{\text {er }}$, il suggère au monarque de poursuivre sa guerre contre la France («Le seul veritable [moyen] s'y trouve en la continuation de la guerre que le Roy de la Grande Bretaigne a entreprise en France» ${ }^{64}$ ), pour inciter les Français à se joindre aux princes du sang dans leur combat contre Richelieu:

(59) Bibliothèque Publique et Universitaire, Genève, manuscrit Tronchin ]62, f. $57 \mathrm{v}^{\circ}$ et 54 (le ms. est mal folioté); B.H.R., LXIV, n² 2,2002, p. 173.

(60) Bibliothèque Publique et Universitaire, Genève, manuscrit Tronchin 148, f. $168 \mathrm{r}^{\circ}$ (inédit).

(61) Se reporter aux citations donnée supra, au sujet de Coutras et d'Ivry.

(62) Samuel Kinser, D’Aubigné and the murder of Concini: "Complaintes du sang du grand Henry", «Studies in Philology», XII, 1965, p. 662695.

(63) Voir par exemple les Tragiques, «Misères», v. 1047-1050.

(64) «Advis au roy de la Grande Bretagne», British Library, Additiona1 Manuscript 48101 (anciennement ms. Yelverton 110), f. 394 r ; B.H.R, LXI-2, 1999, p. 510. 
(...) la France voyant qùe le Cardinal est la cause, ou le pretexte de leurs maux se revoltera contre luy et se joindre volontiers à celuy des Princes du sang qui entreprendra de luy ruyner ${ }^{65}$

L'idée d'exalter la rivalité entre le cardinal et le comte de Soissons n'était pas sotte, à ce point près que Soissons bénéficiait du soutien de l'Empereur et du roi d'Espagne. Le jeu politique d'Agrippa n'aurait donc pas manqué de se retourner contre le parti protestant entier.

Il reste encore beaucoup à dire, car la guerre et l'imaginaire guerrier constituent une clef d'accès privilégiée à l'œuvre d'Agrippa d'Aubigné. On aura moins cherché, dans le présent travail, à évoquer des représentations littéraires, que les combats tout à fait réels, auxquels Agrippa fut mêlé ou sur lesquels il médita. Que cela plaise ou non, ses écrits ne se laissent pas détacher des circonstances qui les ont vu naître. Par le mélauge curieux d'intelligence aiguë et d'esprit de système dont elle témoigne, son œuvre politique et historique apparaît comme représentative de la Renaissance en son déclin et de la difficulté, alors que les Etats-nations acquièrent leur autonomie et que le droit se sécularise, à porter sur la guerre un regard authentiquement moderne, qui ne soit point marqué par des préjugés d'ordre confessionnel.

GILLES BANDERIER

(65) Ibid., British Library, Additional Manuscript 48101 (anciennement ms. Yelverton 110), f. 